\title{
针刀干预对膝骨关节炎兔髌韧带拉伸、蠕变及应力 松驰等生物力学特性的影响
}

\author{
郭长青，张丽萍 \\ 北京中医药大学针炎推拿学院, 北京 100029 \\ E-mail: guochangqing66@163.com \\ 收稿日期: 2016-03-06; 接受日期: 2016-05-17 \\ 国家自然科学基金(批准号: 81173333)资助
}

\begin{abstract}
摘要针刀干预对髌韧带生物力学特性可以产生一定的影响, 故针刀对㮏骨关节炎具有一定的治疗作用. 本 研究采用左后肢伸直位固定制动法造成㮏骨关节炎模型兔(Leporidae), 并应用针刀进行治疗, 治疗结束后, 通过 对㮏关节髌韧带进行应力松驰、蠕变及拉伸等力学测试, 研究针刀干预对咜韧带生物力学的影响. 结果显示, 针 刀干预可以明显改善髌韧带的生物力学特性, 激发髌韧带的拉伸、蠕变、应力松驰特性的恢复, 维护㮏关节的平 衡与稳定.
\end{abstract}

关键词㮏骨关节炎, 针刀, 髌韧带, 生物力学特性

膝骨关节炎(knee osteoarthritis，KOA)是一种人 体易发的慢性、进行性的骨关节病变, 它主要以机体 关节软骨的退变与再修复、软骨下骨的重塑和硬化、 骨赘的形成与出现, 以及滑膜与滕关节腔一系列的 生化反应与形态学变化为特征. 我国最新的流行病 学报道称, 目前膝骨关节炎的发病率越来越高, 且危 害越来越严重 ${ }^{[1]}$. 膝骨关节炎的发病原因与机制目前 还不十分明确, 然而, 从针刀的临床应用中可知, 针 刀松解法可以有效地松解膝关节周围的髌韧带、内外 侧副韧带, 以及局部软组织, 来缓解局部张力, 从而 达到治疗膝骨关节炎的目的 ${ }^{[2]}$. 所以, 膝关节周围韧
带生物力学的变化可能会导致和加剧膝关节应力平 衡的失调状态，而这一平衡的失调则是膝骨关节炎 出现的关键原因. 所以, 通过针刀松解法对膝关节周 围韧带的力学特性加以调节, 从而促进膝关节应力 平衡的调整, 是针刀治疗膝骨关节炎的重要作用途 径. 前期的动物实验已经初步研究了针刀通过松解 膝关节周围软组织达到修复软骨的短期作用机制. 本实验以膝骨关节炎兔 (Leporidae)为研究对象, 观察 针刀干预治疗对膝关节髌㓞带的应力松弛、蠕变及拉 伸等力学特性的影响, 从而研究针刀干预对髌韧带 生物力学的作用.

引用格式: 郭长青, 张丽萍. 针刀干预对膝骨关节炎兔髌韧带拉伸、蠕变及应力松弛等生物力学特性的影响. 中国科学: 生命科学, 2016, 46: 976-982 Guo C Q, Zhang L P. Influences of acupotomy intervention on biomechanical characteristics of tension, creep and stress relaxation of patellar ligament in rabbits with knee osteoarthritis. Sci Sin Vitae, 2016, 46: 976-982, doi: 10.1360/N052016-00015 


\section{1 实验材料与方法}

\section{1 实验动物及分组}

6 月龄新西兰家兔 24 只, 体重 $(2.25 \pm 0.25) \mathrm{kg}$. 由 北京市金牧阳实验动物养殖有限责任公司提供, 在 北京军区总医院骨科研究所动物实验室喂养.

按随机数字表将家兔分为 4 组, 分别是空白组、 模型组、电针组、针刀组, 每组 6 只. 除空白组外, 其 余 3 组均参加造模.

\section{2 主要设备和试剂}

直径 $0.40 \mathrm{~mm}$ 、长 $40 \mathrm{~mm}$ 的汉章牌一次性针刀. 直径 $0.25 \mathrm{~mm}$ 、长 $25 \mathrm{~mm}$ 的环球牌一次性无菌针炎针. LH202H 型 HANS 穴位神经刺激仪(北京华卫产业开 发公司). 激光测量仪 (奥林巴斯公司, 日本). Bose Electro Force 3300 疲劳试验机(BOSE 公司, 美国).

\section{3 膝骨关节炎模型复制}

采用改良后 Videman 法 ${ }^{[3]}$ 即左后肢伸直位固定 制动法: 除空白组外, 另 3 组造模块动物于造模前禁 食 10 16 h, 以 $3 \%$ 龙巴比妥钠溶液按 $30 \mathrm{mg} / \mathrm{kg}$ 剂量 耳缘静脉注射麻醉, 剪去左后肢踝关节以上兔毛, 牵 拉使其处于完全伸直位, 树脂绷带经 $65^{\circ} \mathrm{C} \sim 85^{\circ} \mathrm{C}$ 热水 浸泡软化后, 从腹股沟到趾头固定 (膝关节伸直 $180^{\circ}$, 踝关节背屈 $60^{\circ}$ ), 留出足趾观察血供, 树脂外以石膏 固定, 继用医用纱布包裹, 加以制动并保持其透气 性, 以防止兔撕咬, 共制动 6 周 ${ }^{[3]}$. 如果足趾有坏死、 发黑、浮肿等情况出现, 应立刻拆除石膏、树脂, 待 肿胀消退后再重新固定. 若树脂松动或脱落应及时 重新固定. 在整个造模过程中, 若出现足底溃烂、咬 伤、骨折、皮肤感染、腹泻等情况, 均予以剔除.

\section{4 干预方法}

各组动物处理如下:

( i ) 空白组. 不做任何处理.

(ii) 模型组. 造模后不做干预治疗.

(iii) 电针组. 造模后 1 周, 以 LH202H 型 HANS 穴位神经刺激仪进行电针刺激治疗, 分别连接内膝 眼和外膝眼, 阳陵泉和阴陵泉. 波形疏密波, 频率 $2 / 100 \mathrm{~Hz}$, 强度 $3 \mathrm{~mA}$, 每次 $20 \mathrm{~min}$. 隔天治疗 1 次, 1 周治疗 3 次, 共治疗 3 周.

(iv) 针刀组. 造模后 1 周, 在兔膝关节针刀进针
部位, 以龙胆紫定位, 常规备皮、消毒, 刀口线与髌 韧带纵轴平行, 刀体和髌韧带皮肤切面垂直. 快速刺 入达髌韧带下约 $0.5 \mathrm{~cm}$, 由上而下松解, 出刀并加压 $3 \mathrm{~s}$. 每周 1 次, 共 3 次, 共治疗 3 周.

\section{5 取材}

各组动物在治疗结束 3 周后, 以空气栓塞法处死 进行取材. 股骨于距髌骨上缘 $4 \mathrm{~cm}$ 处钳断, 胫骨于 距髌韧带下缘 $4 \mathrm{~cm}$ 处钳断. 剔除其余组织, 获取髌 骨-髌韧带-胫骨组合体样本, 保持韧带与骨连接处完 好. 将骨-韧带-骨连结的标本用生理盐水浸湿的纱 布包裹, 置于密封袋内密封, $-20^{\circ} \mathrm{C}$ 冰箱保存, 备力学 测试.

\section{6 指标检测}

(1) 拉伸测试. 于测试前一天, 将髌骨-髌㓞带胫骨连结的标本由 $-20^{\circ} \mathrm{C}$ 冰箱转移至 $5^{\circ} \mathrm{C}$ 冰箱中解冻. 测试当天采用游标卡尺测量韧带的长度、宽度, 采用 激光测厚仪测量韧带的厚度, 每条韧带的长度、宽 度、厚度均测量 3 次, 取平均值.

将标本的胫骨外部用牙托粉包埋后, 利用特制 防滑脱夹具安装在 Bose Electro Force 3300 疲劳试验 机的夹头内，标本外部以生理盐水润湿的纱布包裹. 拉伸实验以 $5 \mathrm{~mm} / \mathrm{min}$ 的速度施加应力, 达到最大载 荷, 直至将试件拉断, 实验结束后计算出试样的极限 载荷、最大应力、最大应变、最大位移、弹性模量、 刚度等.

(2) 蠕变测试. 样本处理同前. 将标本的胫骨 外部用牙托粉包埋后，利用特制防滑脱夹具安装在 Bose Electro Force 3300 疲劳试验机的夹头内, 标本 外部以生理盐水润湿的纱布包裹. 以 $5 \mathrm{~mm} / \mathrm{min}$ 的加 载速率、在 $0 \sim 5 \mathrm{Mpa}$ 应力范围内对试件反复加卸载 15 次进行预调处理, 生物材料在应力作用下都有滞 后现象, 在变形过程中有熵的改变, 有机械能损失, 所以每次实验前必须对试样进行预调处理.

正式进行蠕变实验, 以 $0.05 \mathrm{MPa} / \mathrm{s}$ 的应力速度施 加应力, 当试件应力达到 $4 \mathrm{MPa}$ 后, 停止加载, 保持 此应力不变, 计算机程序设定时间从 $\mathrm{t}(0)$ 开始采集数 据, 每 $0.6 \mathrm{~s}$ 采集一个数据, 采集 10 次后, 继续每 $10 \mathrm{~s}$ 采集一个数据, 采集 40 次后, 继续每 $136 \mathrm{~s}$ 采集一个 数据, 采集 50 次后, 共采集 100 个数据, 历时 $7200 \mathrm{~s}$. 达到设定的时间后，记录数据和曲线. 
(3) 应力松弛测试. 样本处理同前. 预调处理 同前. 正式进行应力松驰实验, 以 $0.05 \% / \mathrm{s}$ 的应变速 度施加应变, 当试件长度达到原长的 $5 \%$ 后, 保持此 应变不变, 记录数据, 计算机数据采集程序与蠕变测 试相同.

\section{7 统计处理}

(1) 拉伸测试统计分析. 将力学测试机上的实 验数据导入到 OriginPro 9.0 数据分析软件中, 进行数 据统计分析, 得出每个试件的载荷-位移曲线, 从中 找出试件的极限载荷、最大位移等数据, 拟合出试件 的刚度. 再次利用 OriginPro 9.0 数据分析软件进行数 据分析, 得出每个试件的应力-应变曲线, 从中找出 试件的最大应力、最大应变等数据, 拟合出试件的弹 性模量. 所有数据用 $\bar{x} \pm \mathrm{SD}$ 表示.

(2) 髌韧带蠕变测试统计分析. 假设髌韧带的 初长度为 $L_{0}$, 将试件拉伸至 $L_{1}$, 保持应力不变, 经过 一段时间后, 试件的长度变为 $L_{2}$, 那么: 髌韧带的蠕 变率 $=\left[\left(L_{2} / L_{1}\right)-1\right] \times 100 \%$. 将实验数据整理后, 导入到 SPSS20.0 统计软件中, 用 $\bar{x} \pm \mathrm{SD}$ 表示.

(3) 髌韧带应力松驰测试统计分析. 将试件从 初长度 $L_{0}$ 拉伸至 $L_{1}$ 后, 假设髌韧带的初始应力为 $T_{1}$, 保持此长度不变, 经过一段时间后, 试件的应力变为 $T_{2}$, 那么: 髌韧带的应力松弛率 $=\left(T_{1}-T_{2}\right) / T_{1} \times 100 \%$. 将实验数据整理后, 导入到 SPSS20.0 统计软件中, 用 $\bar{x} \pm \mathrm{SD}$ 表示.

\section{2 结果}

\section{1 髌韧带力学拉伸试验结果}

拉伸结果经统计分析显示, 造模成功后, 模型 组、电针组、针刀组髌韧带的极限载荷明显降低, 与 空白组相比具有极显著性差异 $(P<0.01)$; 干预治疗 后, 针刀组与模型组比较有极显著性差异 $(P<0.01)$, 与空白组比较无显著性差异 $(P>0.05)$; 电针组与空白 组、模型组比较均无显著性差异 $(P>0.05)$; 造模后, 模型组、电针组、针刀组的最大位移与空白组比较有 极显著性差异 $(P<0.01)$; 治疗后, 针刀组与模型组相 比有显著性差异 $(P<0.05)$, 针刀组与空白组比较有显 著性差异 $(P<0.05)$; 电针组与模型组比较无显著性差 异 $(P>0.05)$; 在刚度方面, 各组数据间均无统计学意
义 $(P>0.05)$.

造模后，模型组髌韧带的最大应力、最大应变、 弹性模量均降低, 最大应力与空白组比较有显著性 差异 $(P<0.05)$; 治疗后, 针刀组与电针组的最大应力 与空白组、模型组比较均无显著性差异 $(P>0.05)$; 模 型组最大应变与空白组比较有极显著性差异 $(P<0.01)$; 针刀组最大应变与模型组比较有显著性差 异 $(P<0.05)$, 与空白组比较无显著性差异 $(P>0.05)$; 在弹性模量上, 各组数据间均无统计学意义 $(P>0.05)$ (图 1 3, 表 1 和 2).

\section{2 髌㓞带蠕变测试结果}

各组髌㓞带的蠕变率比较结果：造模后, 模型组 髌韧带的蠕变率略高于空白组的蠕变率，针刀干预 后, 对模型组髌韧带的蠕变率产生了一定的影响, 电

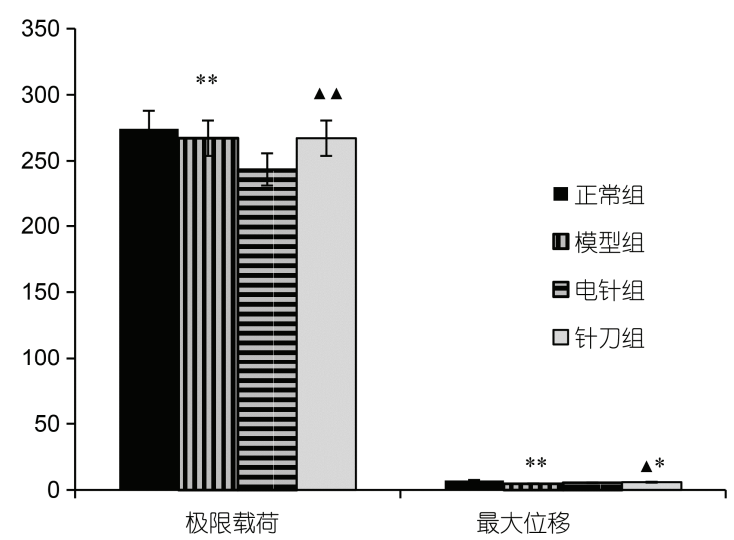

图 1 各组髌㓞带极限载荷、最大位移的比较 与空白组比, **: $P<0.01 ; *: P<0.05 ;$ 与模型组比, $\mathbf{\Lambda} \mathbf{\Lambda}: P<0.01 ; \mathbf{\Lambda}$ $P<0.05$

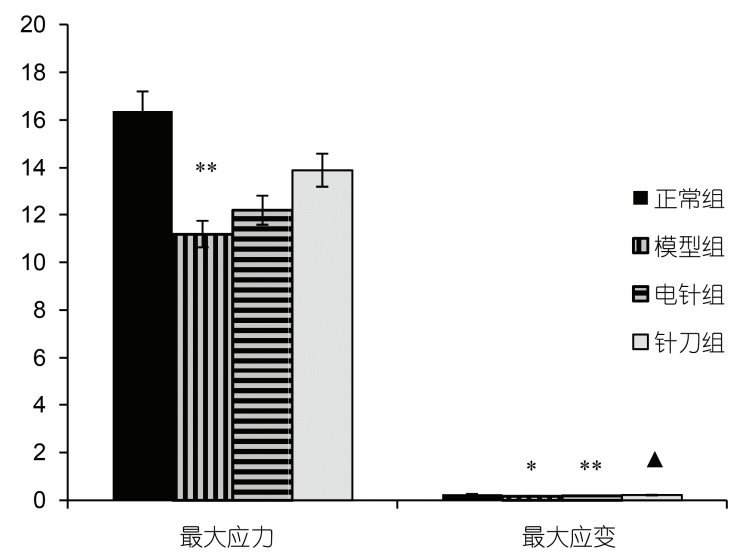

图 2 各组髌韧带最大应力、最大应变的比较 与空白组比, **: $P<0.01 ; *: P<0.05 ;$ 与模型组比, $\mathbf{\Lambda}: P<0.05$ 
表 1 髌韧带极限载荷、最大位移、刚度比较 $(\bar{x} \pm \mathrm{SD})^{\mathrm{a})}$

\begin{tabular}{ccccc}
\hline 组别 & 样本数 $($ 只 $)$ & 极限载荷 $(\mathrm{N})$ & 最大位移 $(\mathrm{mm})$ & 刚度 $(\mathrm{N} / \mathrm{mm})$ \\
\hline 空白组 & 6 & $274.25 \pm 43.02$ & $7.04 \pm 01.01$ & $39.63 \pm 8.37$ \\
模型组 & 6 & $202.32 \pm 46.14^{* *}$ & $4.62 \pm 0.93^{* *}$ & $44.78 \pm 11.38$ \\
电针组 & 6 & $243.53 \pm 25.60$ & $5.47 \pm 0.75$ & $45.71 \pm 11.21$ \\
针刀组 & 6 & $267.18 \pm 35.14^{\mathbf{\Lambda}}$ & $5.86 \pm 0.87^{\mathbf{\Delta}}$ & $46.72 \pm 10.20$ \\
\hline
\end{tabular}

a) 与空白组比, **: $P<0.01 ; *: P<0.05$; 与模型组比,

表 2 髌韧带最大应力、最大应变、弹性模量比较 $(\bar{x} \pm \mathrm{SD})^{\mathrm{a})}$

\begin{tabular}{ccccc}
\hline 组别 & 样本数(只) & 最大应力 $(\mathrm{MPa})$ & 最大应变 $(\%)$ & 弹性模量 $(\mathrm{MPa})$ \\
\hline 空白组 & 6 & $16.38 \pm 4.31$ & $0.25 \pm 0.04$ & $67.31 \pm 22.89$ \\
模型组 & 6 & $11.18 \pm 3.34^{* *}$ & $0.17 \pm 0.03^{*}$ & $65.04 \pm 17.86$ \\
电针组 & 6 & $12.18 \pm 4.47$ & $0.19 \pm 0.03^{* *}$ & $63.91 \pm 14.43$ \\
针刀组 & 6 & $13.9 \pm 4.46$ & $0.22 \pm 0.04^{\mathbf{}}$ & $64.84 \pm 23.64$ \\
\hline
\end{tabular}

a) 与空白组比, **: $P<0.05 ; *: P<0.01$; 与模型组比, $\mathbf{\Lambda}: P<0.05$

针对髌韧带的蠕变率亦有所改善, 但效果不及针刀 组, 说明针刀和电针均对模型的韧带蠕变率有改善 的趋势, 但 4 组间未见显著性差异 $(P>0.05)$ (表 3 和 图 4).

\section{3 髌㓞带应力松驰测试结果}

各组髌㓞带的应力松弛率比较结果, 造模后, 模

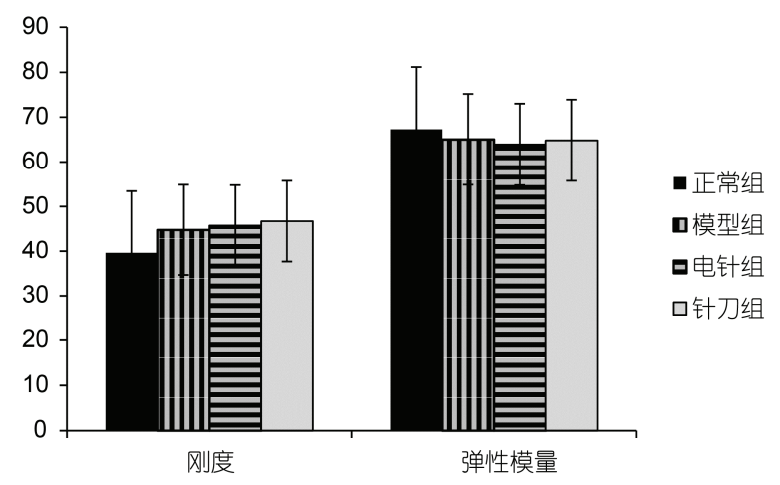

图 3 各组髌韧带刚度、弹性模量的比较 4 组间均无显著性差异, $P>0.05$

表 3 各组髌韧带的蠕变率比较 $(\bar{x} \pm \mathrm{SD})^{\mathrm{a})}$

\begin{tabular}{ccc}
\hline 组别 & 样本数(只) & 蠕变率 $(\%)$ \\
\hline 空白组 & 6 & $0.63 \pm 0.55$ \\
模型组 & 6 & $1.11 \pm 0.31$ \\
电针组 & 6 & $0.94 \pm 0.50$ \\
针刀组 & 6 & $0.72 \pm 0.23$ \\
\hline
\end{tabular}

a) 4 组间均无显著性差异, $P>0.05$

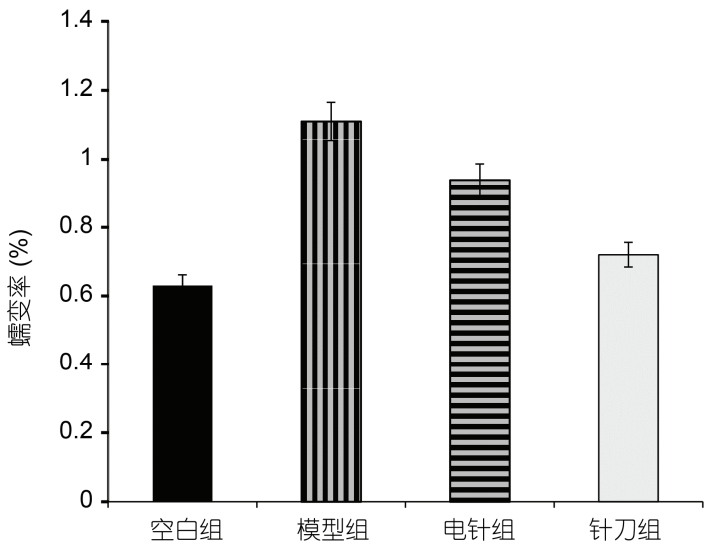

图 4 各组髌韧带的蠕变率比较 4 组间均无显著性差异, $P>0.05$

型组髌㓞带的应力松弛率明显高于空白组的髌韧带 松弛率, 与空白组比较有极显著性差异 $(P<0.01)$; 针 刀干预后, 对模型组韧带的松弛率产生了良好的长 期影响, 与模型组比较有极显著性差异 $(P<0.01)$, 与 空白组比较无显著性差异 $(P>0.05)$; 电针干预后, 髌 韧带的松弛率也有所改善, 与模型组比较有极显著 性差异 $(P<0.01)$, 与空白组比较无显著性差异 $(P>0.05)$, 但从其均值可以看出其效果不及针刀组. 结果显示, 左后肢伸直位固定制动法对髌韧带的松 弛特性产生了影响, 且针刀松解法可以明显改善髌 韧带的松弛特性，电针和针刀均可以改善模型的应 力松弛率，对韧带的应力松弛特性产生不同程度的 影响, 但电针组的改善效果不及针刀组(表 4 和图 5). 


\section{表 4 各组髌韧带的应力松驰率比较 $(\bar{x} \pm \mathrm{SD})^{\mathrm{a})}$}

\begin{tabular}{ccl}
\hline 组别 & 样本数 $($ 只) & 应力松弛率 $(\%)$ \\
\hline 空白组 & 6 & $62.05 \pm 5.27$ \\
模型组 & 6 & $84.3 \pm 10.41^{* *}$ \\
电针组 & 6 & $70.35 \pm 8.39^{\mathbf{\Lambda}}$ \\
针刀组 & 6 & $60.80 \pm 6.46^{\mathbf{\Lambda}}$ \\
\hline
\end{tabular}

a) 与模型组比, $\boldsymbol{\Delta} \mathbf{\Delta}: P<0.01$; 与空白组比, **: $P<0.01$

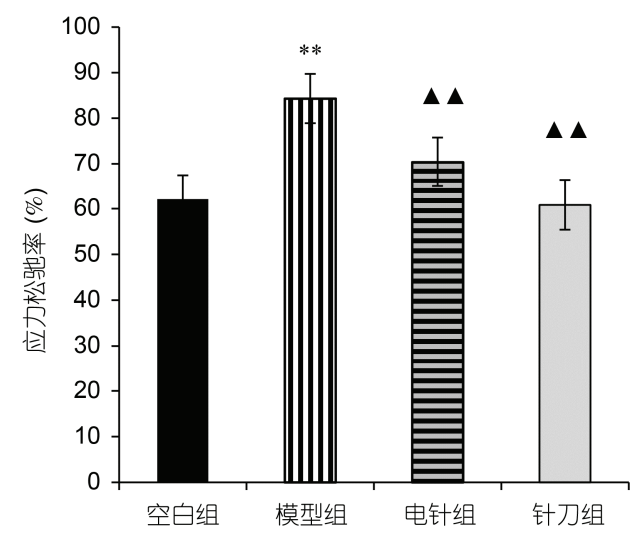

图 5 各组髌韧带的应力松弛率比较

与模型组比, $\mathbf{\Delta} \mathbf{\Delta}: P<0.01$; 与空白组比, $* *: P<0.01$

\section{3 讨论}

本实验采用改良后 Videman 法, 即左后肢伸直 位固定制动法制备膝骨关节炎模型, 改变了膝关节 的力学轴线, 使得关节面负重减低, 造成关节软骨所 承受的力上升, 而膝关节周围的肌肉、韧带等软组织 在长期牵拉的情况下无法收缩, 从而发生痉挛、黏连 与增生, 这又减弱了膝关节的稳定性, 而这些因素都 是发生膝骨关节炎的最初病理变化. 随着造模时间 的延长, 膝关节骨内压力不断增高, 而其关节内部提 供营养的血管之内的血流量逐渐减少, 局部的骨小 梁因为缺乏足够的滋养而发生坏死, 由于骨小梁存 在自身修复功能, 所以当其自我修复之时, 膝关节的 软骨下骨则会出现增生, 进而造成致密组织的生成, 以致膝关节出现一系列的慢性退行性病变. 由此可 见, 应用改良后 Videman 法造成的膝关节病理性损 伤与修复过程和人体膝骨关节炎的发生发展十分相 似. 有文献报道, 在骨性关节炎的发病机制与治疗反 应的研究之中发现, 在实验兔的膝关节于伸直位固 定制动 5 6 周时, 其病理变化与临床上的膝骨关节炎
模型较为符合. 实验兔在膝关节制动 6 周之时, 与正 常组实验兔相比, 表现出眼神黯淡, 对刺激反应出现 迟钝的情况, 日常活动量有所减少, 周身皮毛暗黄、 甚至发生脱落, 饮食不振, 进食量明显减少, 但二便 基本维持正常状态, 且体重有所增加, 小部分模型兔 的膝关节出现活动不利的情况, 甚至不能正常弯曲, 或发生跛行, 触地或者蹬地困难.

膝骨关节炎的发病原因与机制目前还不十分明 确，从膝关节周围韧带损伤与膝骨关节炎的关系来 看, Weiss 和 Maakestad ${ }^{[4]}$ 、王红坡等人 ${ }^{[5]}$ 、杨志发等

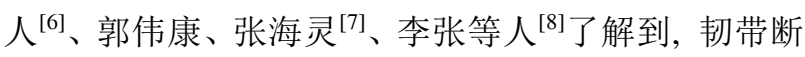
裂对于关节软骨的损伤具有显著的影响, 而在韧带 修复之后，软骨的退行性改变则会出现被抑制的情 况, 且软骨细胞的调亡情况也有所缓解. 从针刀的临 床应用中可知 ${ }^{[9]}$, 针刀松解法可以有效地松解膝关节 周围的髌韧带、内外侧副㓞带, 以及局部软组织, 来 缓解局部张力, 从而达到治疗膝骨关节炎的目的. 所 以, 膝关节周围韧带生物力学的变化可能会导致和 加剧膝关节应力平衡的失调状态, 而这一平衡的失 调则是膝骨关节炎出现的关键原因. 所以, 通过针刀 松解法对膝关节周围韧带的力学特性加以调节, 从 而促进膝关节应力平衡的调整，是针刀治疗膝骨关 节炎的重要作用途径.

临床上，由于各种原因需要进行膝关节制动，而 膝关节制动之后往往会出现一系列的临床表现, 具 体包括膝关节屈伸受到限制, 经常伴随患侧股四头 肌痉挛萎缩, 正常肌力有所降低, 以及膝关节囊与其 周围组织发生黏连、孪缩, 造成膝关节出现僵硬的现 象, 这严重影响着患者的日常生活与工作 ${ }^{[10]}$. 此次实 验采取左后肢伸直固定法进行制动，以制备膝骨关 节炎模型, 观察应用针刀松解法改善膝骨关节炎中 髌韧带生物力学状态的远期疗效, 以探讨针刀治疗 膝骨关节炎的临床疗效，促进膝骨关节炎的治疗.

Jung 等人 ${ }^{[11]}$ 发现, 韧带作为骨与骨之间力传递 的软组织, 属于典型的一种黏弹性材料, 有着拉伸与 黏弹性等两种生物力学特性, 而这些特性被关节制 动所影响着, 而逆转这种不良影响的方法就是解除 制动或者进行运动锻炼. 韧带是一种沿纵轴方向进 行骨与骨之间负荷传导的最适合载体，因此，一般情 况下, 检测韧带的生物力学特性是通过对骨-韧带-骨 这一复合体进行单轴的拉伸实验来进行，而韧带损 
伤之后的力学特性的恢复情况直接反映出了受损韧 带的愈合状态 ${ }^{[12,13]}$. 髌韧带正常范围之内的拉伸、蠕 变、应力松他值能够有效地支持膝关节, 促进膝关节 保持平衡与稳定状态, 而正常范围以外的拉伸、蠕变、 应力松他值却危害着膝关节平衡与稳定状态的维持.

本实验利用针刀进行膝骨关节炎的治疗, 主要 是通过针刀对力学平衡的调整作用, 以打破膝骨关 节炎发生与发展的恶性循环过程, 即通过对膝关节 周围肌肉、㓞带等软组织的调节, 从而达到治疗膝关 节软骨退化的效果. 其机理可能是: 由于机体自身或 者外界的各种刺激因素, 如直接或者间接的暴力伤 害、体重的作用、激素水平的变化等情况, 于膝关节 周围软组织上发生作用, 从而导致膝关节周围肌肉、 㓞带等软组织出现黏连、孪缩等现象, 而这些病理变
化会引起膝关节力学平衡的失调, 这一失调的力学 信号传递至软骨细胞膜之上存在的一氧化氮、整合 素、钻离子等力学信号转导因子, 从而促使软骨组织 之中各条力学转导通路的开放, 从而调控所有通路 中的各种聚集蛋白聚糖、基质金属蛋白酶、蛋白多糖、 各型胶原物质基因与蛋白的表达情况, 从而达到治 疗膝骨关节炎的目的 ${ }^{[14]}$.

由此认为, 针刀松解法通过对膝关节周围软组 织的切割与剥离, 能够达到松解膝关节周围软组织 的黏连与挛缩, 从而激发髌韧带发挥自我修复的功 能, 促进髌韧带生物力学特性的改善与恢复, 并且, 通过与电针对比可以发现, 针刀松解法对于促进膝 骨关节炎髌韧带生物力学恢复的疗效明显优于电针 干预, 值得持续研究与推广.

\section{参考文献}

1 李战武, 王理康. 针刀松解术+功能锻炼对 I 、 II 级膝骨关节炎的疗效. 武警医学, 2014, 25: 290-292

2 金晓飞, 郭长青, 蒋昭霞, 等. 膝骨关节炎模型内侧副韧带的生物力学特性. 中国组织工程研究与临床康复, 2011, 15: 8046-8049

3 Videman T. Experimental osteoarthritis in the rabbit: comparison of different periods of repeated immobilization. Acta Orthop Scand, 2008, 53: 339-347

4 Weiss J A, Maakestad B J. Permeability of human medial collateral ligament in compression transverse to the collagen fiber direction. J Biomech, 2006, 39: 276-283

5 王红坡, 葛英辉, 李玉侠, 等. 正常髌韧带和前交叉韧带的 MRI 测量与分析. 中国运动医学杂志, 2009, 28: 248-250, 259

6 杨志发, 刘展亮, 张惠城. 六股自体腘绳肌腱人字形编织重建膝关节前交叉韧带. 吉林医学, 2015, 36: 960-961

7 张海灵. 前交叉韧带重建修复运动性膝关节损伤: 临床现状与未来. 中国组织工程研究, 2014, 18: 5384-5388

8 李张, 邹国耀, 宋恩鸿. 兔前交叉韧带不同分束断裂对内侧半月板的影响. 新乡医学院学报, 2015, 32: 119-122

9 刘振梅, 裴久国, 张佳. 针刀整体松解术治疗膝关节骨性关节炎临床观察. 湖北中医杂志, 2013, 35: 61-62

10 顾钧青, 郭艳明, 梁永瑛, 等. 近 5 年针刀治疗膝骨关节炎研究概况. 陕西中医, 2013, 34: 1101-1104

11 Jung H J, Fisher M B, Woo S L. Role of biomechanics in the understanding of normal, injured, and healing ligaments and tendons. Sports Med Arthrosc Rehabil Ther Technol, 2009, 1: 9

12 付达尔丽. 针刀与毫针干预对膝骨关节炎兔内、外侧副韧带力学影响的对比研究. 硕士学位论文. 北京: 北京中医药大学, 2014

13 金英利. 针刀对 KOA 兔韧带力学改变及韧带胶原相关因子基因蛋白表达的影响研究. 博士学位论文. 北京: 北京中医药大学, 2014

14 夏鹏李, 雪萍. 骨性关节炎与整合素-FAK 力学信号转导通路的基础研究. 中华临床医师杂志, 2012, 6: 8245-8248 


\title{
Influences of acupotomy intervention on biomechanical characteristics of tension, creep and stress relaxation of patellar ligament in rabbits with knee osteoarthritis
}

\author{
GUO ChangQing \& ZHANG LiPing
}

College of Acupuncture and Moxibustion, Beijing University of Traditional Chinese Medicine, Beijing 100029, China

\begin{abstract}
Acupotomy intervention can impact the biomechanical characteristics of patellar ligament, so it has some effect on knee osteoarthritis. We induced the KOA model by fixing the left legs of the model rabbits and treated them with the method of acupotomy intervention. After the treatment, we tested the tension, creep and stress relaxation data of patellar ligament to study the effect of acupotomy on the biomechanical characteristics of patellar ligament. The results showed that acupotomy intervention can significantly improve the biomechanical properties of patellar ligament, stimulate the recovery of tension, creep, and stress relaxation, and maintain balance and stability of the knee joint.
\end{abstract}

osteoarthritis of the knee, acupotomy, patellar ligament, biomechanical characteristic

doi: 10.1360/N052016-00015 